\title{
Optimal Pump Scheduling Considering Resetting Reservoir to Minimum Level and Rescheduling due to Pump Maintenance
}

\author{
Trust Tawanda* \\ Department of Statistics and Operations Research, National University of Science and Technology, Zimbabwe
}

Submission: January 09, 2018; Published: April 26, 2018

*Corresponding author: Trust Tawanda, Department of Statistics and Operations Research, National University of Science and Technology, P 0 Box AC 939, Ascot, Bulawayo, Zimbabwe, E-mail: trustawanda@gmail.com, n01523351a@students.nust.ac.zw

\begin{abstract}
In this paper we consider two pumping stations, one with three pumps that can be run individually or as combinations and the other with one pump as well as daily maximum volume restriction on water to be pumped out. Night electricity tariffs are lower than day tariffs. Each hour has its constant demand except the $7^{\text {th }}$ hour where demand varies, $7^{\text {th }}$ hour demand variation notice is sent a day before by the water customer. Water from these pumping stations is pumped to a high point service reservoir so that pressure level can be maintained on taps. Service reservoir has a capacity of $10000 \mathrm{~m} 3$ and the water level should not go below $40 \%$ full in order to safeguard supplies in the event of burst water and to meet sudden demands such as firefighting. Linear programming is used to determine which pump combination is to be used during which hour as well as developing optimal schedules when any pump is under maintenance.
\end{abstract}

Keywords: Scheduling; Linear programming; Water pumps; Pump maintenance.

Abbreviations: IP: Integer Programming; MOEA: Multi-objective Evolutionary Algorithms

\section{Introduction}

Significant percentage of energy consumption in the world is used for pumping purposes as a result there is need for pump scheduling with objective of minimizing electricity consumption. Ordan et al. [1] developed a new methodology for optimized real-time operation of a water distribution. The methodology is based on the integration of three models, namely the real-time demand forecasting model, the hydraulic simulation model, and the optimization model. The optimization process is driven by the cost minimization of the energy used for pumping and the maximization of operational reliability. Optimal pump schedules were generated by using a multi-algorithm-genetically-adaptivemethod (AMALGAM), they also performed hydraulic simulations using the EPANET2 model. Lansey and Awumah [2] presented a methodology for determining optimal pump operation schedules for water-distribution systems. In addition to minimizing the energy-consumption cost, their model includes a constraint to limit the number of pumps that are switched on during the planning period. They also adopted a two-level approach whereby the system hydraulics are analyzed in an off-line mode to generate simplified hydraulic and cost functions for an on-line model. These functions developed for each pump combination allow for rapid evaluation within a dynamic programming optimization algorithm.

Racca et al. [3] presented a new optimal pumping scheduling model that integrates the evaporation losses from the reservoirs into the optimization algorithm and provides the optimal pumping policy that minimizes both pumping and water costs. Multi-objective Evolutionary Algorithms (MOEAs) were considered to solve an optimal pump-scheduling problem with four objectives to be minimized, thus electric energy cost, maintenance cost, maximum power peak, and level variation in a reservoir [4]. Wang et al. [5] enhanced genetic algorithm for bi-objective pump scheduling in water supply. Hyeong-Seok et al. [6] applied a binary integer program to optimize pumping schedule of a water supply system in Polonnaruwa, Sri Lanka based on the hourly water demands for the next day. The water demands were forecasted by a combined model consisting of an autoregressive integrated moving average model and an error compensation routine based on exponential smoothing technique. The result showed that the optimization system could reduce the operation cost of the WSS by minimizing electricity for water-pumping; electricity cost for pump operation could be 


\section{Civil Engineering Research Journal}

reduced by $55 \%$. For the past decades, a few forecasting methods have been applied to optimize pumping schedules of WSSs [7]. Genetic algorithm also has been applied for minimization of pumping cost through intermittent water pumping in a WSS [8]. Giacomello et al. considered a hybrid optimization method for effective pump scheduling. They solved the problem by a novel hybrid optimization method that uses linear programing and a greedy algorithm. Naoum-Sawaga et al. [9] proposed new approaches for water pump scheduling and pipe replacement, they also applied a knapsack based heuristic for the leak pipe replacement problem. Jowitt and Germanopolous [10] considered a method based on linear programming for determining optimal schedule of pumping on a 24 hour basis. They considered both unit and maximum demand charge, as well as efficiencies of the available pumps, the structure of the electricity tariff, the customer demand profile, and the hydraulic characteristics and optimization constraints of the network. Sakarya and Mays [11] determined optimal operation of water distribution system pumps with quality considerations. Integer programming (IP) was also applied by Kim et al. [12], Błaszczyk et al. [13] presented the optimal pump scheduling for a large scale water distribution system by applying linear programming to optimize the operating schedule of the pumping system in the Seoul Metropolitan area. They used a multiple regression model to forecast hourly water demands. Bagirov et al. [14] introduced a novel approach for modelling of explicit pump scheduling to minimize energy consumptions by pumps which uses the pump Start/ End run times or continuous variable, and binary integer variables to describe the pump status at the beginning of the scheduling period. Pasha and Lansey [15] linearized pump stations relationships using relationships among energy required, pumping flow, demand factors, tank storage or tank water levels. They formulated a linear programming model and solved for a single tank system for the optimal pump schedule to minimize energy costs. Bragalli et al. [16] proposed a method to design optimal distribution reservoirs and their operation using non-linear programming. Price and Ostfeld [17] examined problem that includes nonlinear convex headloss, leakages and varying total head pump energy consumption constraints. Savic et al. [18] introduces multi-objective Genetic Algorithms (GAs) for pump scheduling in water supply systems. The two objectives considered were minimization of energy and maintenance costs. Pump switching were introduced as a surrogate measure of maintenance cost. The multi-objective algorithm was compared to the single objective GA, with both techniques improved by using hybridization with a local-search method.

\section{Statement of the Problem}

Distribution of potable water requires that water is pumped to reservoir at high point in the system. Pumping uses electricity which has a cost which varies through the day. Considerable saving in electricity costs can be made by choosing when pumping is done but pumping patterns are constrained by the necessity to safeguard supplies. In this case study water is obtained from three different types of source

1. Surface reservoirs,

2. Underground, and

3. Abstraction from rivers.

Water is treated in various ways to make it both safe and acceptable to drink. The treated water is pumped to the reservoir. The service reservoir is situated at high points of the distribution system so that the water pressure is maintained at the taps. Since the service reservoir is at high points of the system it is necessary to pump treated water to this reservoir. Often there are several pumps at the pumping station which are situated at the water treatment works. Various combinations of pumps can be used at the request of the operator. Pumps can be controlled either by switching them on or off. The water companies spend a lot of dollars per annum on electricity of pumping water. Table 1 gives electricity tariff which clearly shows that pumping is cheaper at night than during the day. The flow of water down a pipe is subject to some frictional force from the walls of the pipe which increases at the rate of flow of water increases. Table $2 \&$ 3 gives the power consumption and flow rates for all possible combinations of pumps at Nyamandlovu and Sgodini pumping stations which supplies the Bulawayo reservoir. The Esigodini treatment works can only treat 2000 cubic meters of water per day. The hourly demand for water for the Bulawayo community is given in Table 4. The Bulawayo service reservoir has a capacity of 10000 cubic meters and the water level should not go below $40 \%$ full in order to safeguard supplies in the event of burst water and to meet sudden demands such as for firefighting. A pump schedule is a set of instructions to the operator as to when the pumps at a pump station must be switched on and off. A pump schedule is needed that satisfies demand at the minimum costs while satisfying constraints of the water level in the Bulawayo service reservoir. Pump reschedule is also necessary when a certain pump is under maintenance so as to optimally pump water with the available pumps.

Table 1: An industrial electricity tariff.

\begin{tabular}{|c|c|}
\hline During the daytime $(0700-2000)$ & 5 Cents \\
\hline During the nighttime $(0000-0700)$ & 1.7 Cents \\
\hline
\end{tabular}

Table 2: Pump characteristics.

Output Flow Rate $\left(\mathrm{m}^{3} / \mathrm{h}\right)$

200

Table 3: Pump output flow rate and power consumption.

\begin{tabular}{|c|c|c|}
\hline Pump & $\begin{array}{c}\text { Output Flow Rate } \\
\left(\mathbf{m}^{\mathbf{3}} \mathbf{/ h}\right)\end{array}$ & $\begin{array}{c}\text { Power Consumption } \\
(\mathbf{k W})\end{array}$ \\
\hline A & 300 & 80 \\
\hline B & 100 & 30 \\
\hline A+B & 390 & 110 \\
\hline A+A & 550 & 160 \\
\hline A+A+B & 622 & 190 \\
\hline
\end{tabular}




\section{Civil Engineering Research Journal}

Table 4: Bulawayo community hourly demand (cubic meters).

\begin{tabular}{|c|c|c|c|c|c|c|c|c|c|c|c|c|c|c|c|c|c|c|c|c|}
\hline Hour & $\mathbf{1}$ & $\mathbf{2}$ & $\mathbf{3}$ & $\mathbf{4}$ & $\mathbf{5}$ & $\mathbf{6}$ & $\mathbf{7}$ & $\mathbf{8}$ & $\mathbf{9}$ & $\mathbf{1 0}$ & $\mathbf{1 1}$ & $\mathbf{1 2}$ & $\mathbf{1 3}$ & $\mathbf{1 4}$ & $\mathbf{1 5}$ & $\mathbf{1 6}$ & $\mathbf{1 7}$ & $\mathbf{1 8}$ & $\mathbf{1 9}$ & $\mathbf{2 0}$ \\
\hline $\begin{array}{c}\text { Demand } \\
\text { Scenario }\end{array}$ & & & & & & & $*$ & & & & & & & & & & & & & \\
\hline S.No.1 & 250 & 180 & 150 & 101 & 160 & 230 & 1000 & 980 & 100 & 880 & 720 & 360 & 530 & 530 & 100 & 610 & 790 & 880 & 720 & 3123 \\
\hline S.No.2 & 250 & 180 & 150 & 101 & 160 & 230 & 1200 & 980 & 100 & 880 & 720 & 360 & 530 & 530 & 100 & 610 & 790 & 880 & 720 & 3123 \\
\hline S.No.3 & 250 & 180 & 150 & 101 & 160 & 230 & 1400 & 980 & 100 & 880 & 720 & 360 & 530 & 530 & 100 & 610 & 790 & 880 & 720 & 3123 \\
\hline S.No.4 & 250 & 180 & 150 & 101 & 160 & 230 & 1600 & 980 & 100 & 880 & 720 & 360 & 530 & 530 & 100 & 610 & 790 & 880 & 720 & 3123 \\
\hline S.No.5 & 250 & 180 & 150 & 101 & 160 & 230 & 1800 & 980 & 100 & 880 & 720 & 360 & 530 & 530 & 100 & 610 & 790 & 880 & 720 & 3123 \\
\hline S.No.6 & 250 & 180 & 150 & 101 & 160 & 230 & 2000 & 980 & 100 & 880 & 720 & 360 & 530 & 530 & 100 & 610 & 790 & 880 & 720 & 3123 \\
\hline
\end{tabular}

A unit cost for every kilowatt hour energy that is used is given in the Table 1.

Sgodini Station has 1 pump (Table 2).

Nyamandlovu Station has three pumps, two of type A and one of type B pump which may be run in any combination given in Table 3 below.

\section{Linear Programming}

Linear programming model is modelled in such a way that the solution output indicates minutes in a given hour a pump or pump combination is supposed to be switched on and off during the day and night. The model output is such in a way that it can be used to draw up pump schedules as well as determining the utilization of pumps.

\section{Linear programming model parameters}

$\tau_{0} \rightarrow$ Hourly cost for using pump (A) during the night

$\tau_{1} \rightarrow$ Hourly cost for using pump (A) during the day

$\in_{0} \rightarrow$ Hourly cost for using pump (B) during the night

$\in_{1} \rightarrow$ Hourly cost for using pump (B) during the day

$\gamma_{0} \rightarrow$ Hourly cost for using pump combination $(\mathrm{A}+\mathrm{B})$ during the night

$\gamma_{1} \rightarrow$ Hourly cost for using pump combination $(\mathrm{A}+\mathrm{B})$ during the day

$\delta_{0} \rightarrow$ Hourly cost for using pump combination $(\mathrm{A}+\mathrm{A})$ during the night

$\delta_{1} \rightarrow$ Hourly cost for using pump combination $(\mathrm{A}+\mathrm{A})$ during the day

$\mu_{0} \rightarrow$ Hourly cost for using pump combination $(\mathrm{A}+\mathrm{A}+\mathrm{B})$ during the night

$\mu_{1} \rightarrow$ Hourly cost for using pump combination $(\mathrm{A}+\mathrm{A}+\mathrm{B})$ during the day

$\rho_{0} \rightarrow$ Hourly cost for using pump (SGODINI) during the night

$\rho_{1} \rightarrow$ Hourly cost for using pump (SGODINI) during the day

$D_{K} \rightarrow$ Demand during hour $\mathrm{K}$
$\Theta_{j} \rightarrow$ Output flow rate of pump j in cubic metres per hour, and $\mathrm{j}$

$$
=\text { Pumps }(\mathrm{A}, \mathrm{B}, \mathrm{A}+\mathrm{B}, \mathrm{A}+\mathrm{A}, \mathrm{A}+\mathrm{A}+\mathrm{B}, \mathrm{SGODINI})
$$

$P_{(j) N N K} \rightarrow$ Pump j at Nyamandlovu, during the Night, during hour $\mathrm{K}$, and $\mathrm{j}$

$=$ Pumps $(A, B, A+B, A+A, A+A+B$,

$P_{(j) N D K} \rightarrow$ Pump j at Nyamandlovu, during the Day, during hour $\mathrm{K}$, and $\mathrm{j}$

$=$ Pumps $(\mathrm{A}, \mathrm{B}, \mathrm{A}+\mathrm{B}, \mathrm{A}+\mathrm{A}, \mathrm{A}+\mathrm{A}+\mathrm{B}$,

$P_{(j) S N K} \rightarrow$ Pump j at Sgodini, during the Night, during hour $\mathrm{K}$, and $\mathrm{j}=$ SGODINI pump

$P_{(j) S D K} \rightarrow$ Pump j at Sgodini, during the Day, during hour K, and $\mathrm{j}=$ SGODINI pump

$\boldsymbol{C} \longrightarrow$ Reservoir capacity

$\hat{\otimes}_{S} \rightarrow$ SGODINI pumping station capacity

$\boldsymbol{J}_{K} \rightarrow$ Water accumulated at hour $\mathrm{K}$

\section{Linear programming model}

$\operatorname{MinZ}=\tau_{0} \sum_{K=0}^{7} P_{(A) N N K}+\tau_{1} \sum_{K=8}^{20} P_{(A) N D K}+\epsilon_{0} \sum_{K=0}^{7} P_{(B) N N K}+\epsilon_{1} \sum_{K=8}^{20} P_{(B) N D K}+\gamma_{0} \sum_{K=0}^{7} P_{(A+B) N N K}$

$+\gamma_{1} \sum_{K=8}^{20} P_{(A+B) N D K}+\delta_{0} \sum_{K=0}^{7} P_{(A+A) N N K}+\delta_{1} \sum_{K=8}^{20} P_{(A+A) N D K}+\mu_{0} \sum_{K=0}^{7} P_{(A+A+B) N N K}$

$+\mu_{1} \sum_{K=8}^{20} P_{(A+A+B) N D K}+\rho_{0} \sum_{K=0}^{7} P_{(S) S N K}+\rho_{1} \sum_{K=8}^{20} P_{(S) S D K}$

Constraint set number 1

$P_{(A) N N K}+P_{(B) N N K}+P_{(A+B) N N K}+P_{(A+A) N N K}+P_{(A+A+B) N N K} \leq 1$

$P_{(A) N D K}+P_{(B) N D K}+P_{(A+B) N D K}+P_{(A+A) N D K}+P_{(A+A+B) N D K} \leq 1$

Constraint set number 2

$$
\begin{aligned}
& P_{(S) S N K} \leq 1 \\
& P_{(S) S D K} \leq 1
\end{aligned}
$$

\section{Constraint set number 3}

$$
\left(\Theta_{S}\right) \sum_{K=0}^{7} P_{(S) S N K}+\left(\Theta_{S}\right) \sum_{K=8}^{20} P_{(S) S D K} \leq \hat{\otimes}_{S}
$$




\section{Civil Engineering Research Journal}

\section{Constraint set number 4}

$\left(\Theta_{A}\right) P_{(A) N N K}+\left(\Theta_{B}\right) P_{(B) N N K}+\left(\Theta_{A+B}\right) P_{(A+B) N N K}+\left(\Theta_{A+1}\right) P_{(A+A) N N K}+\left(\Theta_{A+\lambda+B}\right) P_{(A+A+B) N K K}+\left(\Theta_{S}\right) P_{(S) S N K}$ $+\mho_{K-1}-\mho_{K}=D_{K}$

$\left(\Theta_{A}\right) P_{(A) N D K}+\left(\Theta_{B}\right) P_{(B) N D K}+\left(\Theta_{A+B}\right) P_{(A+B) N D K}+\left(\Theta_{A+A}\right) P_{(A+A) N D K}+\left(\Theta_{A+A+B}\right) P_{(A+A+B) N D K}+\left(\Theta_{S}\right) P_{(S) S D K}$ $+\mho_{K-1}-\mho_{K}=D_{K}$

Constraint set number 5

$$
\begin{array}{cl}
\mho_{K} \leq 0.6 \omega & 1 \leq K \leq 19 \\
\mho_{K}=0 & K=20
\end{array}
$$

Results (Table 5 \& 6, Figure 1 \& 2)

Table 6 shows how many minutes a pump is supposed to be switched on in any given pumping hour. It is from this table where pump utilization information is taken from. Optimal scheduling cost at different $7^{\text {th }}$ hour demand scenarios are as follows: 1000 cubic meters (\$110.16), 1200 cubic meters (\$113.36), 1400 cubic meters (\$116.56), and 1600 cubic meters (\$119.76), 1800 cubic meters (\$122.96), and 2000 cubic meters (\$126.84).

Utilization of Pump A decreases as demand level increases, this indicates that Pump A is not economic and optimal to use for long time when demand level is high. Sgodini pump utilization level is uniform even if demand is increasing. Pump combination $(\mathrm{A}+\mathrm{A})$ and $(\mathrm{A}+\mathrm{A}+\mathrm{B})$ increases utilization level as demand increases, this is so because these pump combinations are able to pump high volumes of water to satisfy increasing demand levels as well as minimum reservoir content.

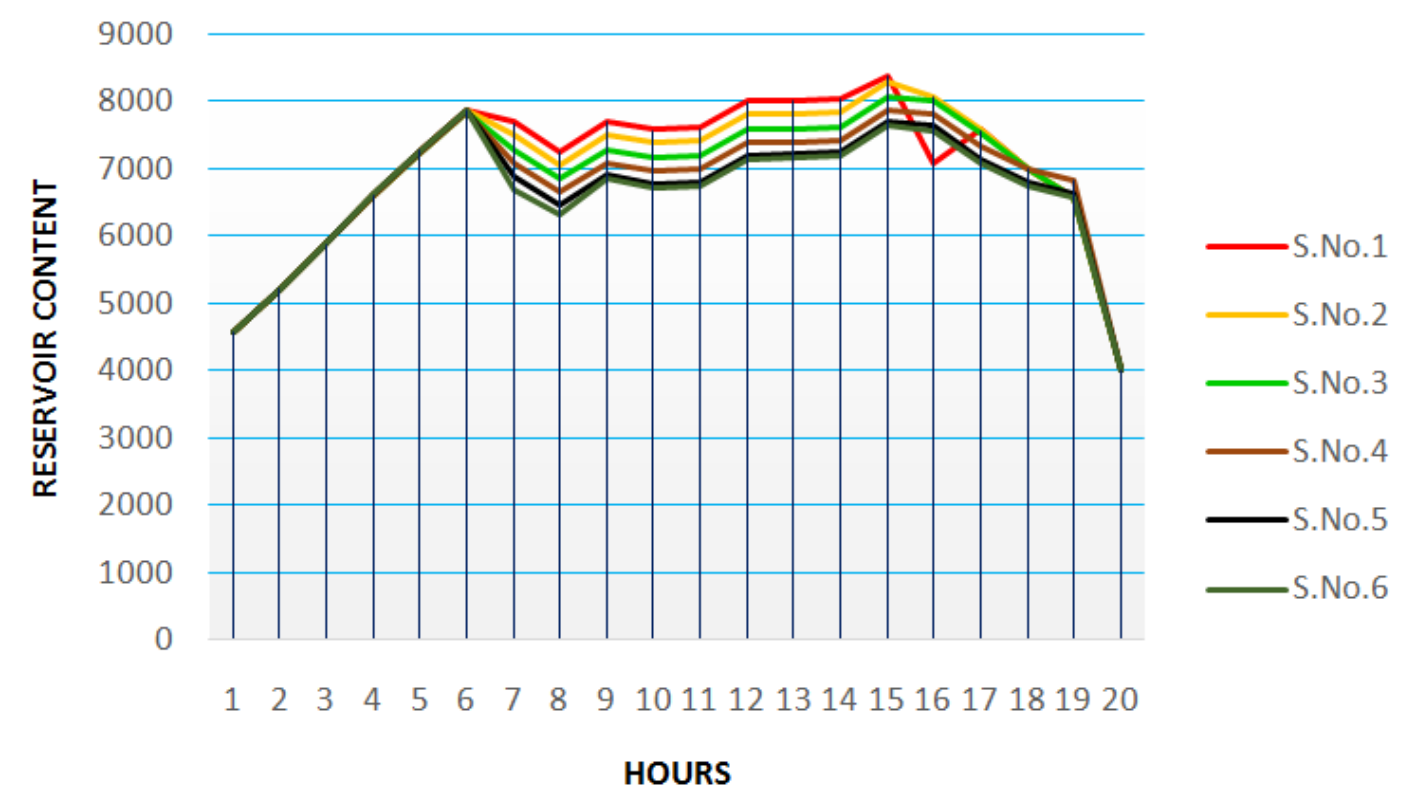

Figure 1: Hourly reservoir Content at different 7th hour demand scenarios

Table 5: Results for hourly reservoir content at different $7^{\text {th }}$ hour demand levels. Hourly levels are all above the minimum reservoir level $(4000$ cubic metres).

\begin{tabular}{|c|c|c|c|c|c|c|c|c|c|c|c|c|c|}
\hline Demand & $\mathbf{1 0 0 0}$ & $\mathbf{1 2 0 0}$ & $\mathbf{1 4 0 0}$ & $\mathbf{1 6 0 0}$ & $\mathbf{1 8 0 0}$ & $\mathbf{2 0 0 0}$ & Demand & $\mathbf{1 0 0 0}$ & $\mathbf{1 2 0 0}$ & $\mathbf{1 4 0 0}$ & $\mathbf{1 6 0 0}$ & $\mathbf{1 8 0 0}$ & $\mathbf{2 0 0 0}$ \\
\hline Hour & $\mathbf{S . N o . 1}$ & $\mathbf{S . N o . 2}$ & $\mathbf{S . N o . 3}$ & $\mathbf{S . N o . 4}$ & $\mathbf{S . N o . 5}$ & $\mathbf{S . N o . 6}$ & $\mathbf{H o u r}$ & $\mathbf{S . N o . 1}$ & $\mathbf{S . N o . 2}$ & $\mathbf{S . N o . 3}$ & S.No.4 & S.No.5 & S.No.6 \\
\hline 1 & 4572 & 4572 & 4572 & 4572 & 4572 & 4572 & 11 & 7603 & 7403 & 7203 & 7003 & 6803 & 6743 \\
\hline 2 & 5214 & 5214 & 5214 & 5214 & 5214 & 5214 & 12 & 7993 & 7793 & 7593 & 7393 & 7193 & 7133 \\
\hline 3 & 5886 & 5886 & 5886 & 5886 & 5886 & 5886 & 13 & 8013 & 7813 & 7613 & 7413 & 7213 & 7153 \\
\hline 4 & 6607 & 6607 & 6607 & 6607 & 6607 & 6607 & 14 & 8033 & 7833 & 7633 & 7433 & 7233 & 7173 \\
\hline 5 & 7269 & 7269 & 7269 & 7269 & 7269 & 7269 & 15 & 8373 & 8283 & 8083 & 7883 & 7683 & 7623 \\
\hline 6 & 7861 & 7861 & 7861 & 7861 & 7861 & 7861 & 16 & 7063 & 8063 & 8023 & 7823 & 7623 & 7563 \\
\hline 7 & 7683 & 7483 & 7283 & 7083 & 6883 & 6683 & 17 & 7573 & 7573 & 7533 & 7333 & 7133 & 7073 \\
\hline 8 & 7253 & 7053 & 6853 & 6653 & 6453 & 6325 & 18 & 6993 & 6993 & 6993 & 7003 & 6803 & 6743 \\
\hline 9 & 7703 & 7503 & 7303 & 7103 & 6903 & 6843 & 19 & 6573 & 6573 & 6573 & 6823 & 6633 & 6573 \\
\hline 10 & 7573 & 7373 & 7173 & 6973 & 6773 & 6713 & 20 & 4000 & 4000 & 4000 & 4000 & 4000 & 4000 \\
\hline
\end{tabular}




\section{Civil Engineering Research Journal}

Table 6: Pump combination schedules at different constant demand levels.

\begin{tabular}{|c|c|c|c|c|c|c|c|c|c|c|c|c|c|c|c|c|c|c|c|c|}
\hline \multicolumn{21}{|c|}{ HOUR } \\
\hline PUMP & 1 & 2 & 3 & 4 & 5 & 6 & 7 & 8 & 9 & 10 & 11 & 12 & 13 & 14 & 15 & 16 & 17 & 18 & 19 & 20 \\
\hline \multicolumn{21}{|c|}{1000} \\
\hline A & OFF & OFF & OFF & OFF & OFF & OFF & OFF & OFF & OFF & OFF & OFF & OFF & OFF & OFF & 26.4 & 60 & 60 & 60 & 60 & OFF \\
\hline B & OFF & OFF & OFF & OFF & OFF & OFF & OFF & OFF & OFF & OFF & OFF & OFF & OFF & OFF & OFF & OFF & OFF & OFF & OFF & OFF \\
\hline$A+B$ & OFF & OFF & OFF & OFF & OFF & OFF & OFF & OFF & OFF & OFF & OFF & OFF & OFF & OFF & OFF & OFF & OFF & OFF & OFF & OFF \\
\hline $\mathrm{A}+\mathrm{A}$ & OFF & OFF & OFF & OFF & OFF & OFF & OFF & 60 & 60 & 60 & 60 & 60 & 60 & 60 & 33.6 & OFF & OFF & OFF & OFF & 60 \\
\hline $\begin{array}{c}\mathrm{A}+\mathrm{A} \\
+\mathrm{B} \\
\end{array}$ & 60 & 60 & 60 & 60 & 60 & 60 & 60 & OFF & OFF & OFF & OFF & OFF & OFF & OFF & OFF & OFF & OFF & OFF & OFF & OFF \\
\hline $\begin{array}{l}\text { SGOD } \\
\text { INI }\end{array}$ & 60 & 60 & 60 & 60 & 60 & 60 & 60 & OFF & OFF & 60 & 60 & 60 & OFF & OFF & OFF & OFF & OFF & OFF & OFF & OFF \\
\hline \multicolumn{21}{|c|}{1200} \\
\hline A & OFF & OFF & OFF & OFF & OFF & OFF & OFF & OFF & OFF & OFF & OFF & OFF & OFF & OFF & OFF & 38.4 & 60 & 60 & 60 & OFF \\
\hline B & OFF & OFF & OFF & OFF & OFF & OFF & OFF & OFF & OFF & OFF & OFF & OFF & OFF & OFF & OFF & OFF & OFF & OFF & OFF & OFF \\
\hline$A+B$ & OFF & OFF & OFF & OFF & OFF & OFF & OFF & OFF & OFF & OFF & OFF & OFF & OFF & OFF & OFF & OFF & OFF & OFF & OFF & OFF \\
\hline$A+A$ & OFF & OFF & OFF & OFF & OFF & OFF & OFF & 60 & 60 & 60 & 60 & 60 & 60 & 60 & 60 & 21.6 & OFF & OFF & OFF & 60 \\
\hline $\begin{array}{c}\mathrm{A}+\mathrm{A} \\
+\mathrm{B}\end{array}$ & 60 & 60 & 60 & 60 & 60 & 60 & 60 & OFF & OFF & OFF & OFF & OFF & OFF & OFF & OFF & OFF & OFF & OFF & OFF & OFF \\
\hline $\begin{array}{l}\text { SGOD } \\
\text { INI }\end{array}$ & 60 & 60 & 60 & 60 & 60 & 60 & 60 & OFF & OFF & 60 & 60 & 60 & OFF & OFF & OFF & OFF & OFF & OFF & OFF & OFF \\
\hline \multicolumn{21}{|c|}{1400} \\
\hline A & OFF & OFF & OFF & OFF & OFF & OFF & OFF & OFF & OFF & OFF & OFF & OFF & OFF & OFF & OFF & OFF & 60 & 50.4 & 60 & $0 \mathrm{FF}$ \\
\hline B & OFF & OFF & OFF & OFF & OFF & OFF & OFF & OFF & OFF & OFF & OFF & OFF & OFF & OFF & OFF & OFF & OFF & OFF & OFF & OFF \\
\hline$A+B$ & OFF & OFF & OFF & OFF & OFF & OFF & OFF & OFF & OFF & OFF & OFF & OFF & OFF & OFF & OFF & OFF & OFF & OFF & OFF & OFF \\
\hline $\mathrm{A}+\mathrm{A}$ & OFF & OFF & OFF & OFF & OFF & OFF & OFF & 60 & 60 & 60 & 60 & 60 & 60 & 60 & 60 & 60 & OFF & 9.6 & OFF & 60 \\
\hline $\begin{array}{c}\mathrm{A}+\mathrm{A} \\
+\mathrm{B}\end{array}$ & 60 & 60 & 60 & 60 & 60 & 60 & 60 & OFF & OFF & OFF & OFF & OFF & OFF & OFF & OFF & OFF & OFF & $\mathrm{OFF}$ & OFF & OFF \\
\hline $\begin{array}{l}\text { SGOD } \\
\text { INI }\end{array}$ & 60 & 60 & 60 & 60 & 60 & 60 & 60 & OFF & OFF & 60 & 60 & 60 & OFF & OFF & OFF & OFF & OFF & OFF & OFF & OFF \\
\hline \multicolumn{21}{|c|}{1600} \\
\hline A & OFF & OFF & OFF & OFF & OFF & OFF & OFF & OFF & OFF & OFF & OFF & OFF & OFF & OFF & OFF & OFF & 60 & OFF & 2.4 & 60 \\
\hline B & OFF & OFF & OFF & OFF & OFF & OFF & OFF & OFF & $\mathrm{OFF}$ & OFF & OFF & OFF & OFF & OFF & OFF & OFF & OFF & OFF & OFF & OFF \\
\hline$A+B$ & OFF & OFF & OFF & OFF & OFF & OFF & OFF & OFF & OFF & OFF & OFF & OFF & OFF & OFF & OFF & OFF & OFF & OFF & OFF & OFF \\
\hline $\mathrm{A}+\mathrm{A}$ & OFF & OFF & OFF & OFF & OFF & OFF & OFF & 60 & 60 & 60 & 60 & 60 & 60 & 60 & 60 & 60 & OFF & 60 & 57.6 & OFF \\
\hline $\begin{array}{c}\mathrm{A}+\mathrm{A} \\
+\mathrm{B}\end{array}$ & 60 & 60 & 60 & 60 & 6 & 60 & 60 & OFF & OFF & OFF & OFF & OFF & OFF & OFF & OFF & OFF & OFF & OFF & OFF & OFF \\
\hline $\begin{array}{l}\text { SGOD } \\
\text { INI }\end{array}$ & 60 & 60 & 60 & 60 & 6 & 60 & 60 & OFF & OFF & 60 & 60 & 60 & OFF & OFF & OFF & OFF & OFF & OFF & OFF & OFF \\
\hline \multicolumn{21}{|c|}{1800} \\
\hline A & OFF & OFF & OFF & OFF & OFF & OFF & OFF & OFF & OFF & OFF & OFF & OFF & OFF & OFF & OFF & OFF & 60 & OFF & OFF & 14.4 \\
\hline B & OFF & OFF & OFF & OFF & OFF & OFF & OFF & OFF & OFF & OFF & OFF & OFF & OFF & OFF & OFF & OFF & OFF & OFF & OFF & OFF \\
\hline$A+B$ & OFF & OFF & OFF & OFF & OFF & OFF & OFF & OFF & OFF & OFF & OFF & OFF & OFF & OFF & OFF & OFF & OFF & OFF & OFF & OFF \\
\hline $\mathrm{A}+\mathrm{A}$ & OFF & OFF & OFF & OFF & OFF & OFF & OFF & 60 & 60 & 60 & 60 & 60 & 60 & 60 & 60 & 60 & OFF & 60 & 60 & 45.6 \\
\hline $\begin{array}{c}\mathrm{A}+\mathrm{A} \\
+\mathrm{B}\end{array}$ & 60 & 60 & 60 & 60 & 60 & 60 & 60 & OFF & OFF & OFF & OFF & OFF & OFF & OFF & OFF & OFF & OFF & OFF & OFF & OFF \\
\hline $\begin{array}{l}\text { SGOD } \\
\text { INI }\end{array}$ & 60 & 60 & 60 & 60 & 60 & 60 & 60 & OFF & OFF & 60 & 60 & 60 & OFF & OFF & OFF & OFF & OFF & OFF & OFF & OFF \\
\hline
\end{tabular}




\section{Civil Engineering Research Journal}

\begin{tabular}{|c|c|c|c|c|c|c|c|c|c|c|c|c|c|c|c|c|c|c|c|c|}
\hline \multicolumn{10}{|c|}{2000} \\
\hline A & OFF & OFF & OFF & OFF & OFF & OFF & OFF & OFF & OFF & OFF & OFF & OFF & OFF & OFF & OFF & OFF & 60 & OFF & OFF & OFF \\
\hline B & OFF & OFF & OFF & OFF & OFF & OFF & OFF & OFF & OFF & OFF & OFF & OFF & OFF & OFF & OFF & OFF & OFF & OFF & OFF & OFF \\
\hline A+B & OFF & OFF & OFF & OFF & OFF & OFF & OFF & OFF & OFF & OFF & OFF & OFF & OFF & OFF & OFF & OFF & OFF & OFF & OFF & OFF \\
\hline A+A & OFF & OFF & OFF & OFF & OFF & OFF & OFF & OFF & 3.6 & 60 & 60 & 60 & 60 & 60 & 60 & 60 & OFF & 60 & 60 & 60 \\
\hline $\begin{array}{c}\text { A+A } \\
+B\end{array}$ & 60 & 60 & 60 & 60 & 60 & 60 & 60 & 60 & 56.4 & OFF & OFF & OFF & OFF & OFF & OFF & OFF & OFF & OFF & OFF & OFF \\
\hline $\begin{array}{c}\text { SGOD } \\
\text { INI }\end{array}$ & 60 & 60 & 60 & 60 & 60 & 60 & 60 & OFF & OFF & 60 & 60 & 60 & OFF & OFF & OFF & OFF & OFF & OFF & OFF & OFF \\
\hline
\end{tabular}

\section{PUMP COMBINATION UTILIZATION}

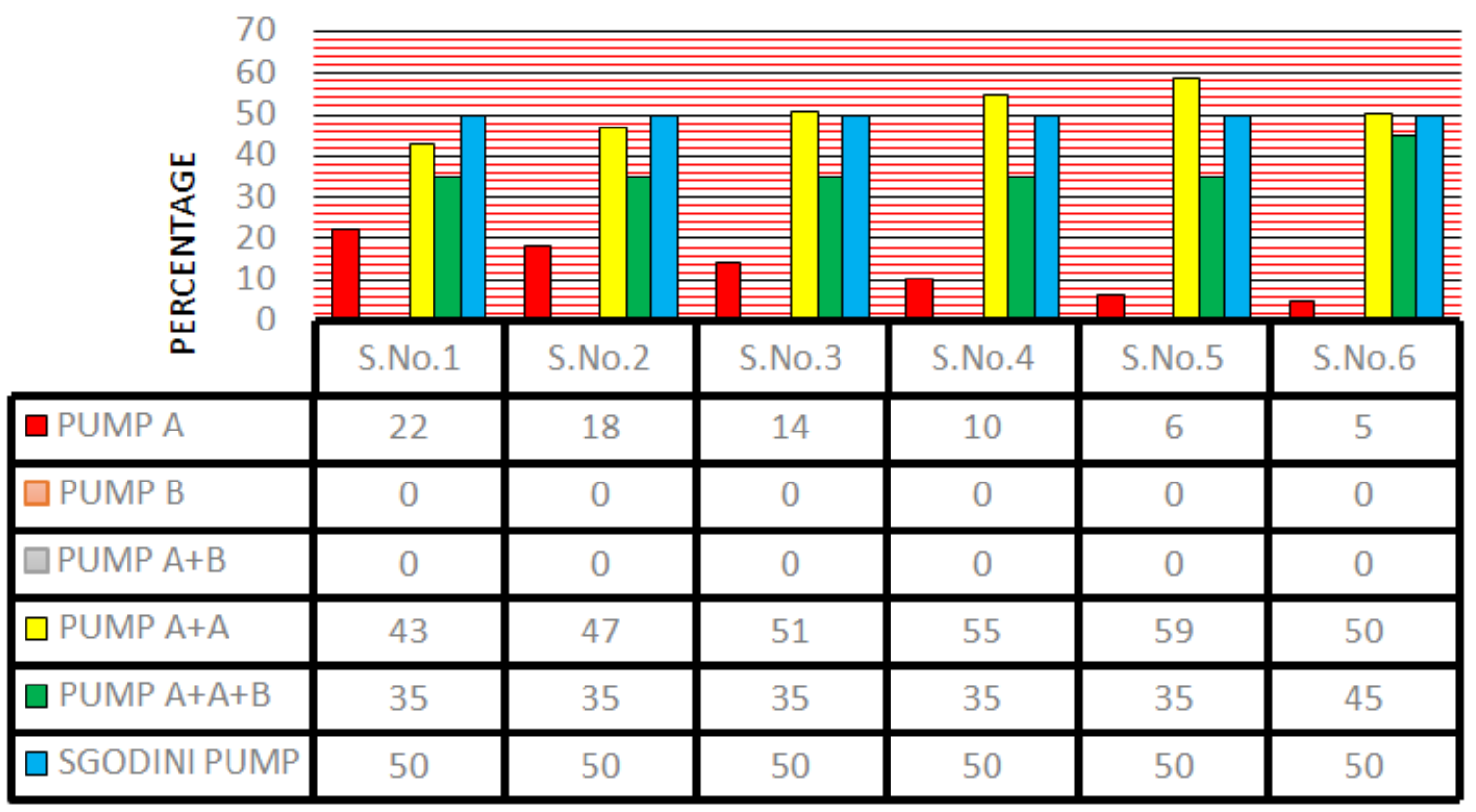

Figure 2: Pump combination utilization when all pumps are in working condition.

\section{Pump Rescheduling}

We assume that at most one pump is under maintenance in any given day. The unavailability of any given pump due to maintenance doesn't only affect the total number of pumps available, but it also reduce the total number of pump

\section{Assuming that pump B is under maintenance}

Table 7: Hourly reservoir content at different demand levels when pump B is under maintenance.

\begin{tabular}{|c|c|c|c|c|c|c|c|c|c|c|c|c|c|}
\hline Demand & 1000 & 1200 & 1400 & 1600 & 1800 & 2000 & Demand & 1000 & 1200 & 1400 & 1600 & 1800 & 2000 \\
\hline Hour & S.No.1 & S.No. 2 & S.No.3 & S.No.4 & S.No.5 & S.No.6 & Hour & S.No.1 & S.No.2 & S.No.3 & S.No.4 & S.No.5 & S.No.6 \\
\hline 1 & 4500 & 4500 & 4500 & 4500 & $\infty$ & $\infty$ & 11 & 7489 & 6899 & 6699 & 6499 & $\infty$ & $\infty$ \\
\hline 2 & 5070 & 5070 & 5070 & 5070 & $\infty$ & $\infty$ & 12 & 7489 & 7289 & 7089 & 6889 & $\infty$ & $\infty$ \\
\hline 3 & 5670 & 5670 & 5670 & 5670 & $\infty$ & $\infty$ & 13 & 7509 & 7309 & 7109 & 6909 & $\infty$ & $\infty$ \\
\hline 4 & 6319 & 6319 & 6319 & 6319 & $\infty$ & $\infty$ & 14 & 7529 & 7329 & 7129 & 6929 & $\infty$ & $\infty$ \\
\hline 5 & 6909 & 6909 & 6909 & 6909 & $\infty$ & $\infty$ & 15 & 7979 & 7779 & 7579 & 7379 & $\infty$ & $\infty$ \\
\hline 6 & 7429 & 7429 & 7429 & 7429 & $\infty$ & $\infty$ & 16 & 7919 & 7719 & 7519 & 7319 & $\infty$ & $\infty$ \\
\hline 7 & 7179 & 6979 & 6779 & 6579 & $\infty$ & $\infty$ & 17 & 7429 & 7229 & 7073 & 7073 & $\infty$ & $\infty$ \\
\hline
\end{tabular}




\section{Civil Engineering Research Journal}

\begin{tabular}{|c|c|c|c|c|c|c|c|c|c|c|c|c|c|}
\hline 8 & 6749 & 6549 & 6349 & 6149 & $\infty$ & $\infty$ & 18 & 7099 & 6899 & 6743 & 6743 & $\infty$ & $\infty$ \\
\hline 9 & 7199 & 6999 & 6799 & 6599 & $\infty$ & $\infty$ & 19 & 6823 & 6729 & 6573 & 6573 & $\infty$ & $\infty$ \\
\hline 10 & 7069 & 6869 & 6669 & 6469 & $\infty$ & $\infty$ & 20 & 4000 & 4000 & 4000 & 4000 & $\infty$ & $\infty$ \\
\hline
\end{tabular}

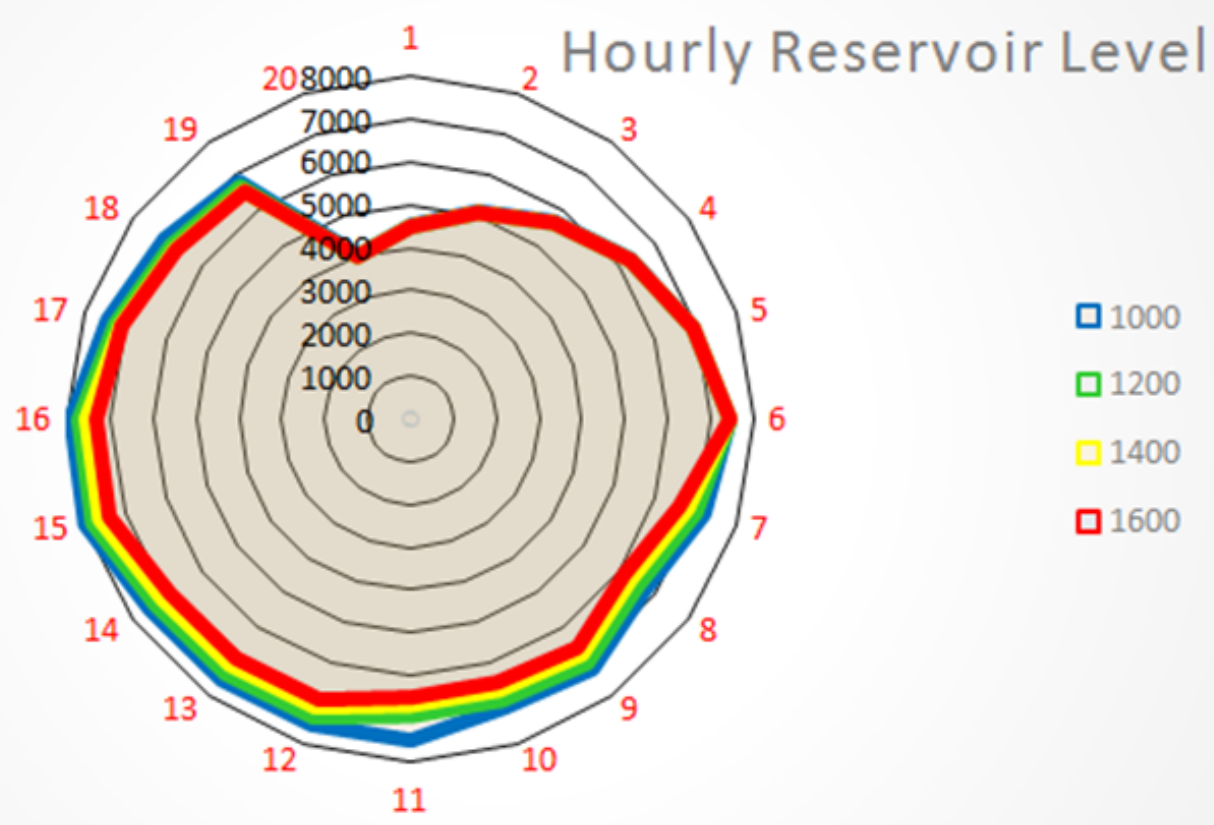

Figure 3: Hourly reservoir content when pump B is under maintenance.

When pump B is under maintenance, we remain with three pumps to be scheduled, thus pump A, A+A, and Sgodini pump. These pumps when scheduled using linear programming the solution lies within the infeasible region when demand is above 1600 cubic meters (Table 7, Figure 3).

When pump $B$ is under repair, we have three pump combinations to be scheduled thus, Pump A, Pump A+A, and Sgodini pump. These pumps were scheduled in such a way that minimum reservoir content was not violated as well as

Table 8: Pump combination schedules at different demand levels when Pump B is under maintenance.

\begin{tabular}{|c|c|c|c|c|c|c|c|c|c|c|c|c|c|c|c|c|c|c|c|c|}
\hline \multicolumn{21}{|c|}{ HOUR } \\
\hline PUMP & 1 & 2 & 3 & 4 & 5 & 6 & 7 & 8 & 9 & 10 & 11 & 12 & 13 & 14 & 15 & 16 & 17 & 18 & 19 & 20 \\
\hline \multicolumn{21}{|c|}{1000} \\
\hline A & OFF & OFF & OFF & OFF & OFF & OFF & OFF & OFF & OFF & OFF & OFF & OFF & OFF & OFF & OFF & OFF & 60 & OFF & 24 & 60 \\
\hline $\mathrm{A}+\mathrm{A}$ & 60 & 60 & 60 & 60 & 60 & 60 & 60 & 60 & 60 & 60 & 60 & 60 & 60 & 60 & 60 & 60 & OFF & 60 & 36 & OFF \\
\hline $\begin{array}{l}\text { SGOD } \\
\text { INI }\end{array}$ & 60 & 60 & 60 & 60 & 60 & 60 & 60 & OFF & OFF & 60 & 60 & 60 & OFF & OFF & OFF & OFF & OFF & OFF & OFF & OFF \\
\hline \multicolumn{21}{|c|}{1200} \\
\hline A & OFF & OFF & OFF & OFF & OFF & OFF & OFF & OFF & OFF & OFF & OFF & OFF & OFF & OFF & OFF & OFF & 60 & OFF & OFF & 36 \\
\hline$A+A$ & 60 & 60 & 60 & 60 & 60 & 60 & 60 & 60 & 60 & 60 & 60 & 60 & 60 & 60 & 60 & 60 & OFF & 60 & 60 & 24 \\
\hline $\begin{array}{l}\text { SGOD } \\
\text { INI }\end{array}$ & 60 & 60 & 60 & 60 & 60 & 60 & 60 & OFF & OFF & 60 & 60 & 60 & OFF & OFF & OFF & OFF & OFF & OFF & OFF & OFF \\
\hline
\end{tabular}

meeting demand requirement. When Pump B is under repair or maintenance these pumps combinations can only pump a maximum $7^{\text {th }}$ hour demand of 1600 cubic metres. In general when pump B is not working we cannot supply a demand above 1600 when using available pumps. Optimal scheduling cost at different 7th hour demand scenarios are as follows: 1000 cubic meters (\$114.65), 1200 cubic meters (\$117.85), 1400 cubic meters (\$121.76), and 1600 cubic meters (\$128.56) (Table 8, Figure 4). 


\section{Civil Engineering Research Journal}

\begin{tabular}{|c|c|c|c|c|c|c|c|c|c|c|c|c|c|c|c|c|c|c|c|c|}
\hline \multicolumn{10}{|c|}{1400} \\
\hline A & OFF & OFF & OFF & OFF & OFF & OFF & OFF & OFF & OFF & OFF & OFF & OFF & OFF & OFF & OFF & OFF & 48 & OFF & OFF & OFF \\
\hline A+A & 60 & 60 & 60 & 60 & 60 & 60 & 60 & 60 & 60 & 60 & 60 & 60 & 60 & 60 & 60 & 60 & 12 & 60 & 60 & 60 \\
\hline $\begin{array}{c}\text { SGOD } \\
\text { INI }\end{array}$ & 60 & 60 & 60 & 60 & 60 & 60 & 60 & OFF & OFF & 60 & 60 & 60 & OFF & OFF & OFF & OFF & OFF & OFF & OFF & OFF \\
\hline \multicolumn{10}{|c|}{10} & 1600 \\
\hline A & OFF & OFF & OFF & OFF & OFF & OFF & OFF & OFF & OFF & OFF & OFF & OFF & OFF & OFF & OFF & OFF & 1 & OFF & OFF & OFF \\
\hline A+A & 60 & 60 & 60 & 60 & 60 & 60 & 60 & 60 & 60 & 60 & 60 & 60 & 60 & 60 & 60 & 60 & 59 & 60 & 60 & 60 \\
\hline $\begin{array}{c}\text { SGOD } \\
\text { INI }\end{array}$ & 60 & 60 & 60 & 60 & 60 & 60 & 60 & OFF & OFF & 60 & 60 & 60 & OFF & OFF & OFF & OFF & OFF & OFF & OFF & OFF \\
\hline
\end{tabular}

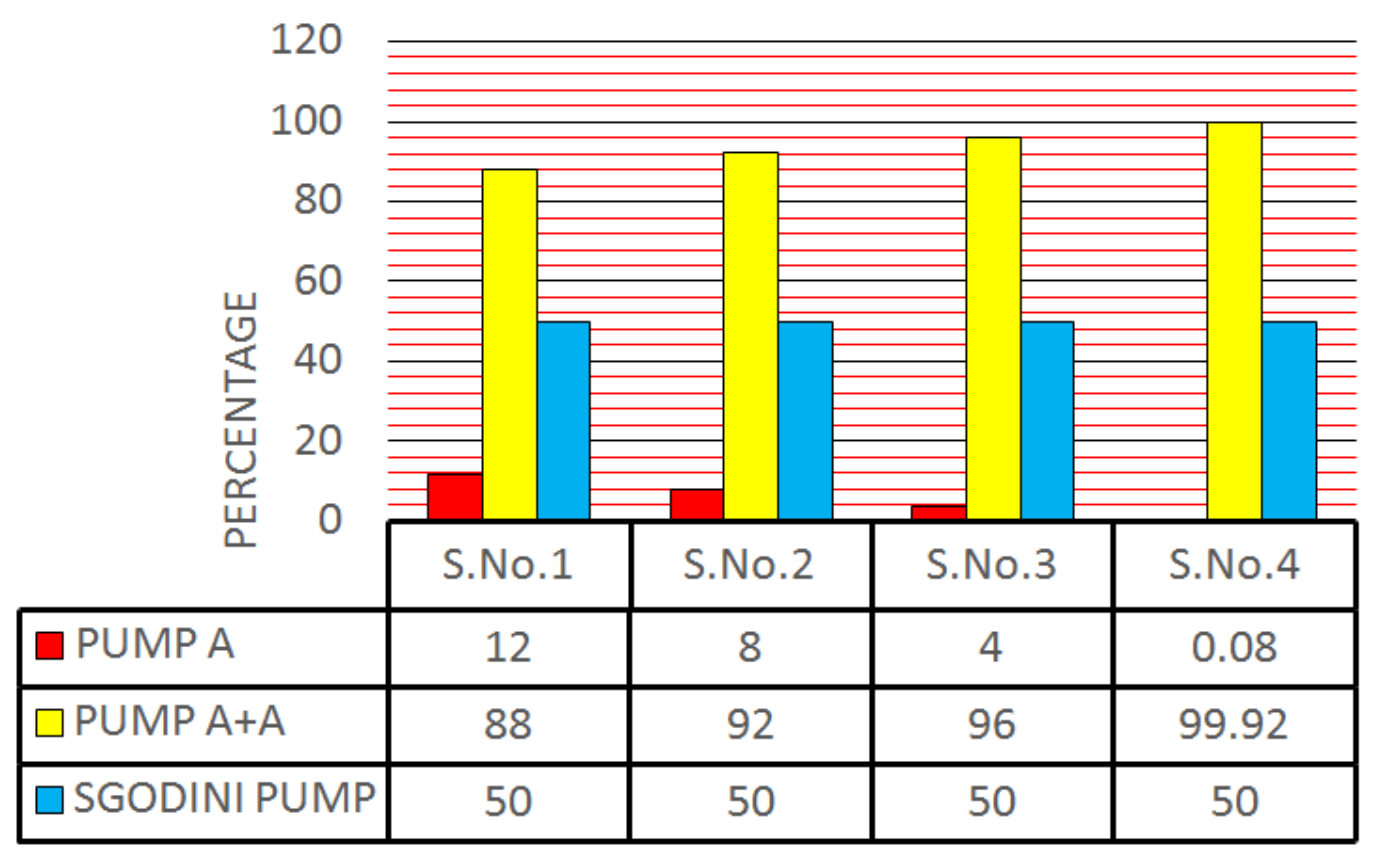

Figure 4: Pump combination utilization when pump B is under maintenance.

Pump $(\mathrm{A}+\mathrm{A})$ utilization factor as demand level increases. When demand level is at 1600 cubic metres pump $(A+A)$ has to work almost throughout the day, with $99.92 \%$ utilization, and pump A is utilized for $0.08 \%$, and this implies that pump A is not capable, optimal or economic when demand level increases. Utilization of Sgodini pump is uniform throughout deferent demand levels (50\%).

\section{Assuming that sgodini pump is under maintenance}

When Sgodini pump is under maintenance, pumps $\mathrm{A}, \mathrm{B}, \mathrm{A}+\mathrm{A}$, $A+B, A+A+B$ are the ones available for rescheduling. The optimal schedule of these pumps cannot supply demand capacity above 1000 cubic meters. The optimal cost for pumping when $7^{\text {th }}$ hour demand is 1000 cubic meters is $\$ 144.75$ (Table 9 \& 10, Figure $5 \& 6)$.

Table 9: Hourly reservoir content at different demand levels when Sgodini pump is under maintenance.

\begin{tabular}{|c|c|c|c|c|c|c|c|c|c|c|c|c|c|}
\hline Demand & 1000 & 1200 & 1400 & 1600 & 1800 & 2000 & Demand & 1000 & 1200 & 1400 & 1600 & 1800 & 2000 \\
\hline Hour & S.No.1 & S.No.2 & S.No.3 & S.No.4 & S.No.5 & S.No.6 & Hour & S.No.1 & S.No.2 & S.No.3 & S.No.4 & S.No.5 & S.No.6 \\
\hline 1 & 4372 & $\infty$ & $\infty$ & $\infty$ & $\infty$ & $\infty$ & 11 & 6091 & $\infty$ & $\infty$ & $\infty$ & $\infty$ & $\infty$ \\
\hline 2 & 4814 & $\infty$ & $\infty$ & $\infty$ & $\infty$ & $\infty$ & 12 & 6353 & $\infty$ & $\infty$ & $\infty$ & $\infty$ & $\infty$ \\
\hline 3 & 5286 & $\infty$ & $\infty$ & $\infty$ & $\infty$ & $\infty$ & 13 & 6445 & $\infty$ & $\infty$ & $\infty$ & $\infty$ & $\infty$ \\
\hline 4 & 5807 & $\infty$ & $\infty$ & $\infty$ & $\infty$ & $\infty$ & 14 & 6537 & $\infty$ & $\infty$ & $\infty$ & $\infty$ & $\infty$ \\
\hline 5 & 6269 & $\infty$ & $\infty$ & $\infty$ & $\infty$ & $\infty$ & 15 & 7059 & $\infty$ & $\infty$ & $\infty$ & $\infty$ & $\infty$ \\
\hline 6 & 6661 & $\infty$ & $\infty$ & $\infty$ & $\infty$ & $\infty$ & 16 & 7071 & $\infty$ & $\infty$ & $\infty$ & $\infty$ & $\infty$ \\
\hline 7 & 6283 & $\infty$ & $\infty$ & $\infty$ & $\infty$ & $\infty$ & 17 & 6857 & $\infty$ & $\infty$ & $\infty$ & $\infty$ & $\infty$ \\
\hline
\end{tabular}




\section{Civil Engineering Research Journal}

\begin{tabular}{|c|c|c|c|c|c|c|c|c|c|c|c|c|c|}
\hline 8 & 5925 & $\infty$ & $\infty$ & $\infty$ & $\infty$ & $\infty$ & 18 & 6599 & $\infty$ & $\infty$ & $\infty$ & $\infty$ & $\infty$ \\
\hline 9 & 6447 & $\infty$ & $\infty$ & $\infty$ & $\infty$ & $\infty$ & 19 & 6501 & $\infty$ & $\infty$ & $\infty$ & $\infty$ & $\infty$ \\
\hline 10 & 6189 & $\infty$ & $\infty$ & $\infty$ & $\infty$ & $\infty$ & 20 & 4000 & $\infty$ & $\infty$ & $\infty$ & $\infty$ & $\infty$ \\
\hline
\end{tabular}

Table 10: Pump combination schedules at different demand levels when Sgodini pump is under maintenance.

\begin{tabular}{|c|c|c|c|c|c|c|c|c|c|c|c|c|c|c|c|c|c|c|c|c|}
\hline \multicolumn{21}{|c|}{ HOUR } \\
\hline PUMP & 1 & 2 & 3 & 4 & 5 & 6 & 7 & 8 & 9 & 10 & 11 & 12 & 13 & 14 & 15 & 16 & 17 & 18 & 19 & 20 \\
\hline \multicolumn{21}{|c|}{1000} \\
\hline A & OFF & OFF & OFF & OFF & OFF & OFF & OFF & OFF & OFF & OFF & OFF & OFF & OFF & OFF & OFF & OFF & 8.6 & OFF & OFF & OFF \\
\hline B & OFF & OFF & OFF & OFF & OFF & OFF & OFF & OFF & OFF & OFF & OFF & OFF & OFF & OFF & OFF & OFF & OFF & OFF & OFF & OFF \\
\hline$A+B$ & OFF & OFF & OFF & OFF & OFF & OFF & OFF & OFF & OFF & OFF & OFF & OFF & OFF & OFF & OFF & OFF & OFF & OFF & OFF & OFF \\
\hline $\mathrm{A}+\mathrm{A}$ & OFF & OFF & OFF & OFF & OFF & OFF & OFF & OFF & OFF & OFF & OFF & OFF & OFF & OFF & OFF & OFF & OFF & OFF & OFF & OFF \\
\hline $\begin{array}{c}\mathrm{A}+\mathrm{A} \\
+\mathrm{B}\end{array}$ & 60 & 60 & 60 & 60 & 60 & 60 & 60 & 60 & 60 & 60 & 60 & 60 & 60 & 60 & 60 & 60 & 51.4 & 60 & 60 & 60 \\
\hline
\end{tabular}

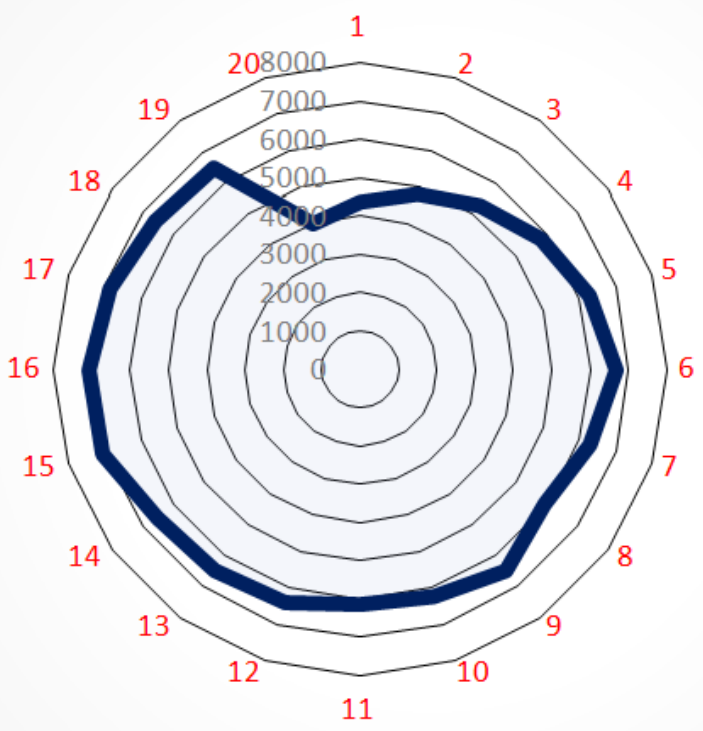

$\mathbf{\square} 1000$

Figure 5: Hourly reservoir content when Sgodini pump is under maintenance.

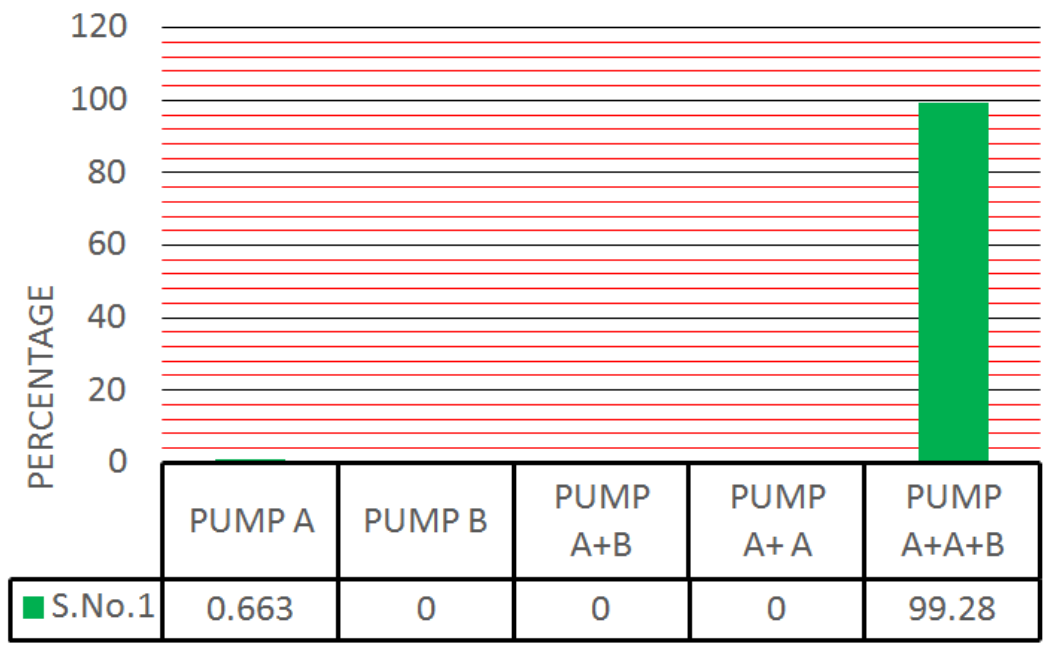

Figure 6: combination utilization when Sgodini pump is under maintenance 
When Sgodini pump is under maintenance we can only supply a minimum and a maximum of 1000 cubic metres. The utilization of pumps are as follows, Pump A (\%0.663), Pump B (\%0), Pump $A+B(\% 0)$, Pump A+A (\%0) and Pump A+A+B (\%99.28). This indicates that Sgodini pump in important pump type when supplying levels of demand more than 1000 cubic metres. On the other hand this reveals that Pump B, Pump A+B, and Pump A+B are dependent of Sgodini pump. When Sgodini pump is under maintenance, any demand level more than 1000 cubic meters lies within the infeasible region.

\section{Assuming that pump $A$ is under maintenance}

When pump A is under maintenance, pumps B and Sgodini are the only ones available for rescheduling. The solution for scheduling these pumps lies in the infeasible region. This implies that they are not capable of supplying the $7^{\text {th }}$ hour demand levels.

\section{Conclusion}

We have significantly demonstrated how linear programming can be used to optimize pumping costs as well as scheduling 1600 pump combinations. Since we can supply a maximum of cubic meters when pump B is under maintenance, a maximum cubic metres when Sgodini pump is under maintenance, 100 of and a maximum of zero cubic meteres when pump $\mathrm{A}$ is under maintenance, this indicates that pump A is very useful to meet cubic meters and more. Pump B is useful 1000 demand levels of cubic metres, 1600 to supply demand level which is more than pump Sgodini is useful for supplying demand level which is more cubic metres. In general if the pumping company needs 1000 than to invest in buying new pumps it has to buy pumps of type A and Sgodini pump type. Furthermore, the results have shown that Pumps $(B, A+B, A+A)$ are not economic or capable when Sgodini pump is under repair, this is evidenced by their utilization of .zero percent when Sgodini pump is under maintenance

\section{Appendix}

Linear programming model with all pumps in LINGO format.

This model was edited through eliminating a pump under maintenance from the model to schedule the available pumps 7 th hour demand can be varied in this model thus .optimally cubic meters) to analyze $2000,1800,1600,1400,1200$ ) .the solutions at deferent demand levels

MIN =

1.36*PANN1+1.36*PANN2+1.36*PANN3+1.36*PAN-

N $4+1.36$ * PANN $5+1.36$ * PANN 6+ 1.36*PANN $7+4$ *PAND $8+$

$4 *$ PAND9+4*PAND10+4*PAND11+4*PAND12+4*PAND13+4*PAN D14+4*PAND15+4*PAND16+4*PAND18+

$4 *$ PAND19+4*PAND20+0.50*PBNN1+0.50*PBNN2+0.50*PBNN3+0.50*PBNN4+0.50*PBNN5+0.50*PBNN6+

$0.50 *$ PBNN7+1.50*PBND8+1.50*PBND9+1.50*PB-

ND10+1.50*PBND11+1.50*PBND12+1.50*PB-
ND13+1.50*PBND14+1.50*PBND15+1.50*PBND16+1.50*PBND17+1.50*PBND18+1.50*PBND19+1.50*PBND20+1.87*PAB$\mathrm{NN} 1+$

1.87*PABNN2+1.87*PABNN3+1.87*PABNN4+1.87*PABN$\mathrm{N} 5+1.87$ * $\mathrm{PABNN} 6+1.87$ * PABN $7+5.50 *$ PABN D $8+$

5.50*PABND9+5.50*PABND10+5.50*PABND11+5.50*PAB$\mathrm{ND} 12+5.50 * \mathrm{PABND} 13+5.50 * \mathrm{PABND} 14+5.50 * \mathrm{PAB}-$ $\mathrm{ND} 15+5.50 * \mathrm{PABND} 16+5.50 * \mathrm{PABND} 17+5.50 * \mathrm{PAB}-$ ND18+5.50*PABND19+5.50*PABND20+2.72*PAAN$\mathrm{N} 1+2.72 *$ PAANN $2+$

$2.72 *$ PAANN3+2.72*PAANN4+2.72*PAANN5+2.72*PAANN6+2.72*PAANN7+8*PAAND8+8*PAAND9+8*PAAND10+8*PAAND11+8*PAAND12+8*PAAND13+8*PAAND 14+8*PAAND 15+8*PAAND16+8*PAAND17+8*PAAND 18+ 8*PAAND19+8*PAAND20+3.23*PAABNN1+3.23*PAABN$\mathrm{N} 2+3.23 *$ PAABNN3+3.23*PAABNN 4+3.23*PAABNN $5+$

3.23*PAABNN6+3.23*PAABNN7+9.50*PAABND8+9.50*PAAB$\mathrm{ND} 9+9.50 * \mathrm{PAABND} 10+9.50 * \mathrm{PAABND} 11+\quad 9.50 * \mathrm{PAAB}-$ ND12+9.50*PAABND13+9.50*PAABND14+9.50*PAABND $15+9.50 *$ PAABND $16+9.50 *$ PAABND $17+$

9.50*PAABND18+9.50*PAABND19+9.50*PAABND20+0.33*PSSN1+0.33*PSSN2+0.33*PSSN3+0.33*PSSN4+ $0.33 *$ PSSN $5+0.33 *$ PSSN6+0.33*PSSN 7+1*PSSD8+ $1 *$ PSSD9+1*PSSD10+1*PSSD11+1*PSSD12+1*PSSD13+ $1 *$ PSSD14+1*PSSD15+1*PSSD16+1*PSSD17+1*PSSD18+1*PSSD $19+1 *$ PSSD 20 ;

PANN1+PBNN1+PABNN1+PAANN1+PAABNN1<=1; PANN2+PBNN2+PABNN2+PAANN2+PAABNN2<=1; PANN3+PBNN3+PABNN3+PAANN3+PAABNN3<=1; PANN4+PBNN4+PABNN4+PAANN4+PAABNN4<=1; PANN5+PBNN5+PABNN5+PAANN5+PAABNN5<=1; PANN6+PBNN6+PABNN6+PAANN6+PAABNN6<=1; PANN7+PBNN7+PABNN7+PAANN7+PAABNN7<=1; PAND8+PBND8+PABND8+PAAND8+PAABND8<=1; PAND9+PBND9+PABND9+PAAND9+PAABND9<=1; PAND10+PBND10+PABND10+PAAND10+PAABND10<=1; PAND11+PBND11+PABND11+PAAND11+PAABND11<=1; PAND12+PBND12+PABND12+PAAND12+PAABND12<=1; PAND13+PBND13+PABND13+PAAND13+PAABND13<=1; PAND14+PBND14+PABND14+PAAND14+PAABND14<=1; PAND15+PBND15+PABND15+PAAND15+PAABND15<=1; PAND16+PBND16+PABND16+PAAND16+PAABND16<=1; 


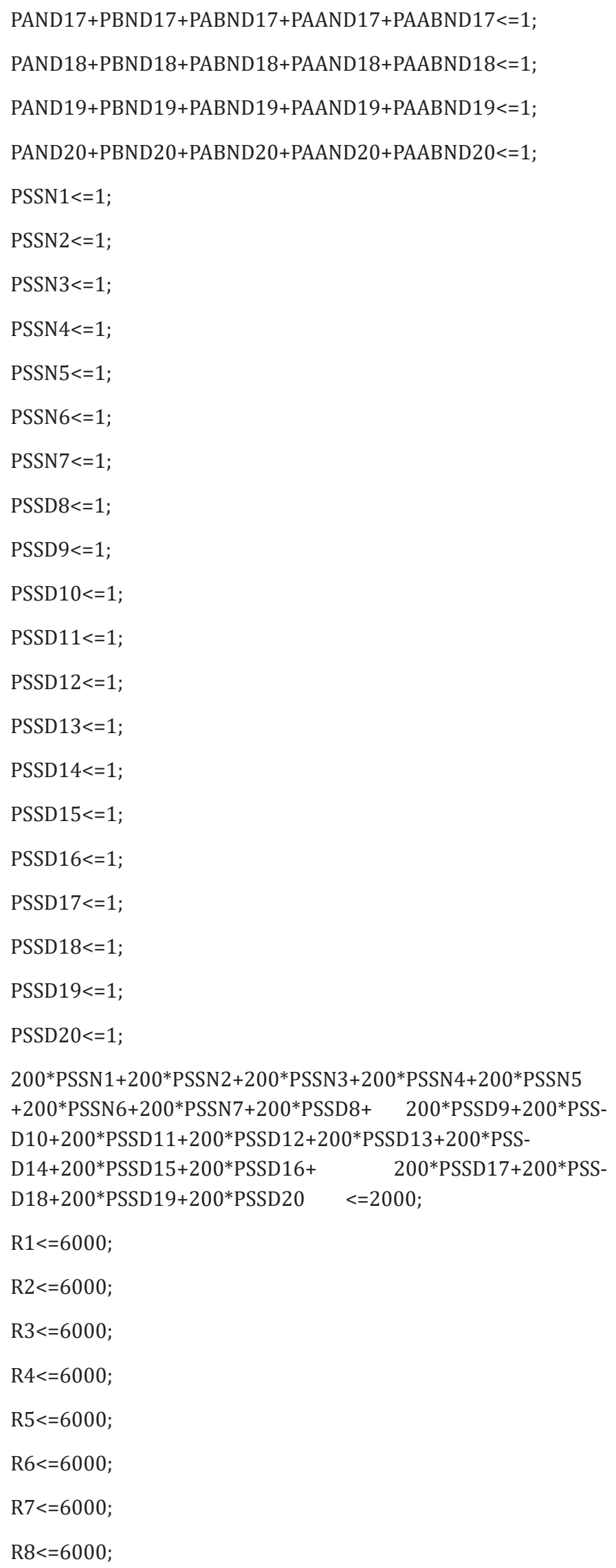

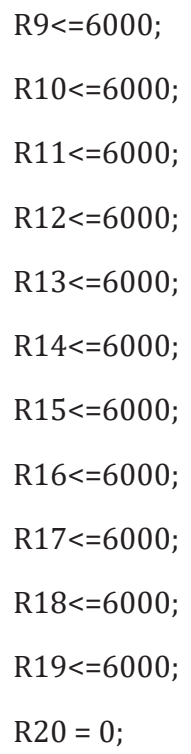




\section{Civil Engineering Research Journal}

D $15+622 *$ PAABND $15+200 *$ PSSD $15+\mathrm{R} 14-\mathrm{R} 15=100$;

$300 *$ PAND16+100*PBND16+390*PABND16+550*PAAN -

D 16+622*PAABND16+200*PSSD 16+R15-R16=610;

$300 *$ PAND17+100*PBND17+390*PABND17+550*PAAN-

$\mathrm{D} 17+622 * \mathrm{PAABND} 17+200 * \mathrm{PSSD} 17+\mathrm{R} 16-\mathrm{R} 17=790$;

300*PAND18+100*PBND18+390*PABND18+550*PAAN-

D $18+622 *$ PAABND18+200*PSSD 18+R17-R18=880;

300*PAND19+100*PBND19+390*PABND19+550*PAAN-

D19+622*PAABND19+200*PSSD19+R18-R19=720;

300*PAND20+100*PBND20+390*PABND20+550*PAAN-

D $20+622 *$ PAABND $20+200 *$ PSSD $20+$ R $19-\mathrm{R} 20=3123$;

\section{References}

1. Odan F, Ribeiro Reis L, Kapelan Z (2015) Real-Time Multi-objective Optimization of Operation of Water Supply Systems. Journal of Water Resources Planning and Management 141(9).

2. Lansey K, Awumah K (1994) Optimal Pump Operations Considering Pump Switches. Journal of Water Resources Planning and Management 120(1): 17-35.

3. Recaa J, García-Manzano , Martínez J (2015) Optimal pumping scheduling model considering reservoir evaporation. Agricultural Water Management 148: 250-257.

4. Baran B, Lucken C, Sotelo A (2005) Multi-objective pump scheduling optimization using evolutionary strategies. Advances in Engineering Software 36(1): 39 -47.

5. Wang J, Chang T, Chen J (2009) An enhanced genetic algorithm for bi-objective pump scheduling in water supply, Experts Systems with applications 36(7): 10249-10258.

6. Hyeong-Seok K, Hyunook K, Jaekyeong L, Ingyu L, Byoung-Youn K, et al. (2014) Optimization of pumping schedule based on water demand forecasting using combined model of autoregressive integrated moving average and exponential smoothing. Water Science \& Technology Water Supply 15(1): 188-195.

7. Adamowski J, Fung Chan H, Prasher SO, Ozga-Zielinski B, Sliusarieva A (2012) Comparison of multiple linear and nonlinear regression, autoregressive integrated moving average, artificial neural network, and wavelet artificial neural network methods for urban water demand forecasting in Montreal, Canada. Water Resources Research 48(1): W01528.

8. Wang JY, Chen FG, Chen JS (2013) A green pump scheduling algorithm for minimizing power consumption and land depletion. Concurrent Engineering 21(2): 121-128.

9. Naoum-Sawaya J, Ghaddar B, Arandia E, Erc B (2015) Simulation - Optimization approaches for water pump scheduling and pipe replacement problems. European Journal of operations research 246(1): 293-306.

10. Jowitt P, Germanopoulous G (1992) Optimal pump scheduling in water supply networks, Journal of water resources planning and management 118(4): 406-422.

11. Sakarya A, Mays L (2000) Optimal operation of water distribution pumps considering water quality, Journal of water resources planning and management 126(4): 210-220.

12. Kim SG, Koo JY, Kim HY, Choi YJ (2007) Optimization of pumping schedule based on forecasting the hourly water demand in Seoul. Water Science and Technology: Water Supply 7(5-6): 85-93.

13. Błaszczyk J, Karbowski A, Krawczyk K, Malinowski K, Allidina A (2012) Optimal pump scheduling for large scale water transmission system by linear programming. Journal of Telecommunications \& Information Technology 3: 91-96.

14. Bagirov AM, Barton AF, Mal-Jetmarova H, AL Nuaimat A, Ahmed ST, et al. (2013) An algorithm for minimization of pumping costs in water distribution systems using a novel approach to pump scheduling. Mathematical and computer modelling 53(3-4): 873 -886.

15. Pasha M, Lansey K (2009) Optimal scheduling by linear programming. World environmental and water resources congress, p. 1-10.

16. Bragalli C, D'Ambrosio C, Lee J, Lodi A, Toth P (2012) On the optimal design of water distribution networks: a practical MINLP approach. Optimization and Engineering 13(2): 219-246.

17. Price E, Ostfeld A (2004) Descrete pump scheduling and leakage control using linear programming for optimal operation of water distribution systems. Journal of hydraulic engineering 140(6).

18. Savic DA, Walters GA, Schwab M (2005) Multiobjective genetic algorithms for pump scheduling in water supply. Lecture Notes in Computer Science: Evolutionary Computing 1305: 227-223.

\section{Your next submission with Juniper Publishers will reach you the below assets}

- Quality Editorial service

- Swift Peer Review

- Reprints availability

- E-prints Service

- Manuscript Podcast for convenient understanding

- Global attainment for your research

- Manuscript accessibility in different formats

( Pdf, E-pub, Full Text, Audio)

- Unceasing customer service

Track the below URL for one-step submission https://juniperpublishers.com/online-submission.php 